\title{
ESTRUCTURAS ESPACIALES DE BARRAS DESPLEGABLES
}

\author{
(SPACIAL STRUCTURES OF EXPANDABLE FRAMES)
}

\section{Félix Escrig, Dr. Arquitecto}

Escuela Técnica Superior de Arquitectura de Sevilla

Departamento de Estructuras

\section{RESUMEN}

A partir de estructuras desplegables planas muy simples, como pueden ser las aspas articuladas en su punto medio, se plantea una serie sucesiva de combinaciones espaciales de complejidad creciente que mantienen las mismas condiciones de plegabilidad que los elementos de partida. Mediante una metodología razonado de ensamblaje, paralela a la utilizada en estructuras espaciales de barras convencionales, se definen distintos tipos de estructuras desplegables en una clasificación que pretende ser completa y que se resume en cuatro tipos básicos.

Estos tipos, a su vez, pueden tener un crecimiento longitudinal superficial o espacial, multiplicando el número de posibilidades formales asi como el de aplicaciones.

Si las aspas iniciales tienen algún tipo de irregularidad, articulación excéntrica, brazos de distinta longitud, más de una articulación intermedia o son distintas entre ellas, se produce una distorsion de los sistemas generados anteriormente que dará lugar a formas de gran utilidad arquitectónica. En este caso los elementos deberán cumplir unas condiciones de compatibilidad para que el plegado sea posible.

Se exponen también algunas soluciones constructivas de encuentros y nudos asi como de mecanismos automáticos de plegado y desplegado.

Finalmente se hace una referencia muy elemental al modo de trabajo de estas estructuras y a los esfuerzos que se introducen en sus barras.

\section{Introducción}

Vamos a partir de un elemento plano formado por varias aspas consecutivas (Fig. 1).

Este elemento es deformable sin más que apretarlo o estirarlo de sus extremos y cambia de forma aumentado su. altura cuando disminuye su longitud y viceversa (Fig. 2).

Es lo que en resistencia de materiales se llama un mecanismo, y su utilización como estructura está restringida por su movilidad que lo hace inestable frente a cargas aplicadas.

Pero supongamos que mediante una barra adicional o alguna coacción exterior fijamos la altura « $\mathrm{H} »$ o la longi-

\section{SUMMARY}

From simple expandable plane structures, as the articulated crosses at their midpoint could be, we have established a succession of spacial combinations of growing complexity, with the same conditions of foldability as the original elements. By means of a rational methodology of joints, similar to those used in conventional spatial frame structures, several types of expandable structures had been defined in a classification which intends to be complete and could be resumed in four basical types.

These types, moreover, could have a linear, a plane or a spatial growing, increasing formal possibilities and practical uses.

If original crosses have any irregularity as excentric joints, struts of different length, more than one intermediate joint or of different size, a distortion of the generated systems, reffered above, is produced, which should give us shapes of huge architectural utility. In these cases crosses must be of compatible length to make possible the folding process.

Some constructive solutions for joints and for folding and expanding mechanisms are also shown.

Finally, we have introduced a short reference to the working way of these structures as well as the stresses acting in their struts.

tud "L" (Figs. 3 y 4). En este caso, mediante esfuerzos axiles y flectores en las barras, este elemento es capaz de transmitir a los apoyos cargas que se apliquen en puntos intermedios.

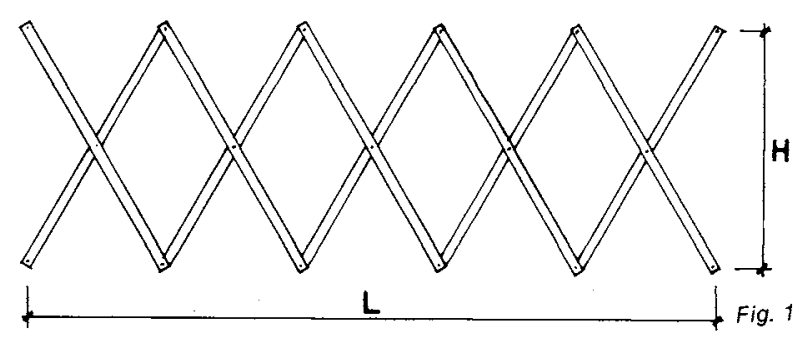



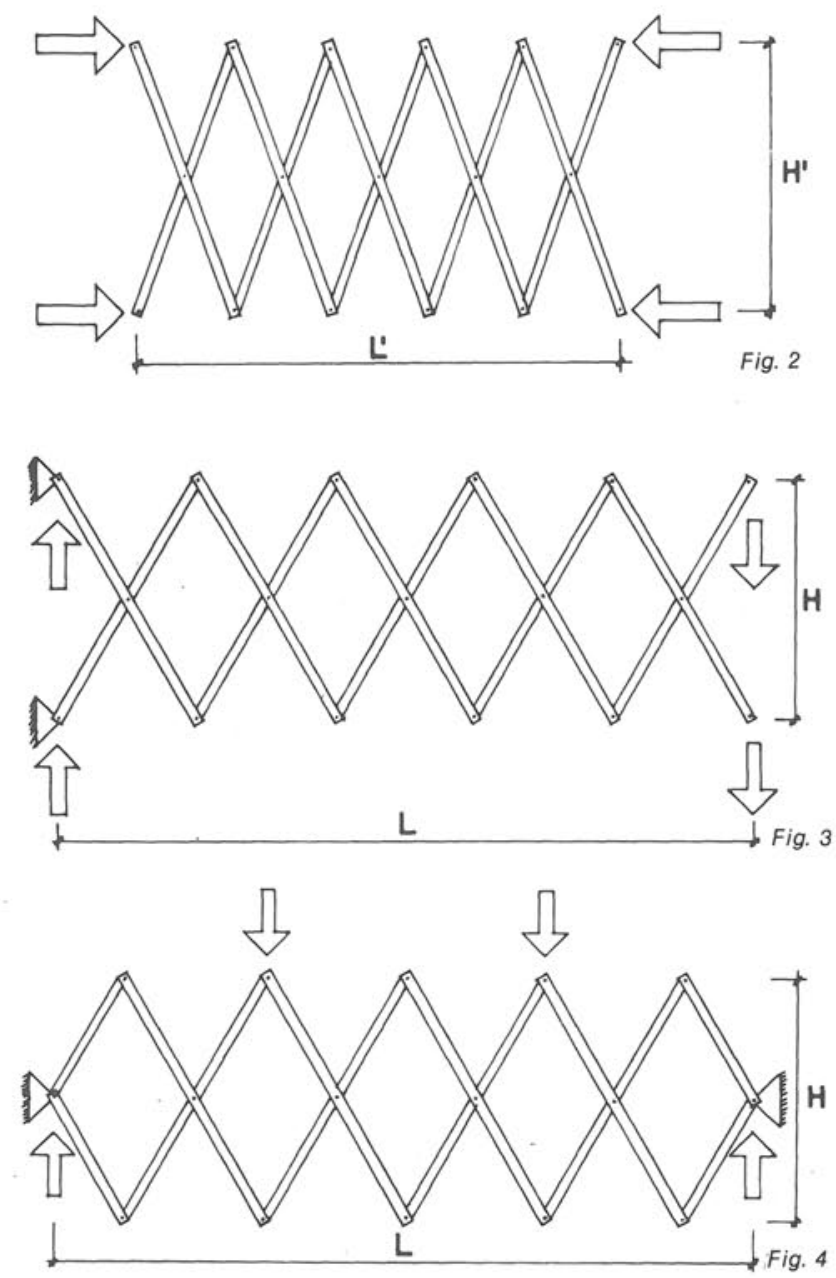
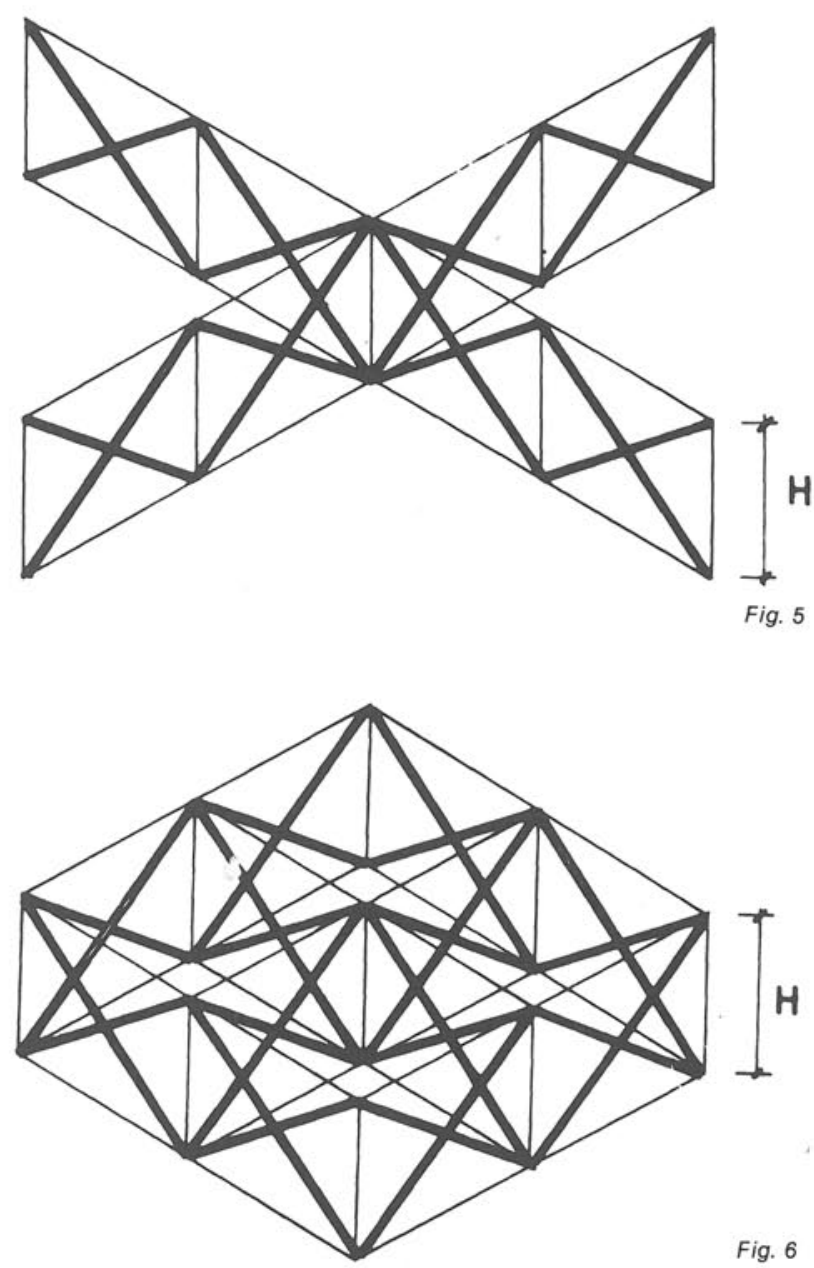

Esta estructura es bien conocida y no requiere más explicación puesto que es utilizada en numerosas aplicaciones prácticas.

\section{Generación de estructuras básicas}

Supongamos ahora un conjunto formado por dos elementos como éstos que se crucen perpendicularmente (Fig. 5). Si deformamos uno de ellos estaremos forzando la deformación del otro y sólo con variar la altura "H» el grupo de barras se encogerá o expandirá.

Aprovechando esta colaboración vamos a construir un conjunto en que se ensamblen muchos de estos elementos planos (Fig. 6). Por la misma razón anterior en cuanto variemos una dimensión todo el conjunto se verá arrastrado a tomar una disposición más compacta o más abierta. Hemos construido una estructura espacial de barras.

El conjunto puede ser muy complejo como se advierte en las figuras 7,8 y 9 que ilustra las fotografias de una estructura real generada como se ha explicado.

Si en lugar de cruzar los elementos perpendicularmente lo hacemos de tres en tres formando ángulos de $60^{\circ}$, el comportamiento será similar (Fig. 10) y variando una di-

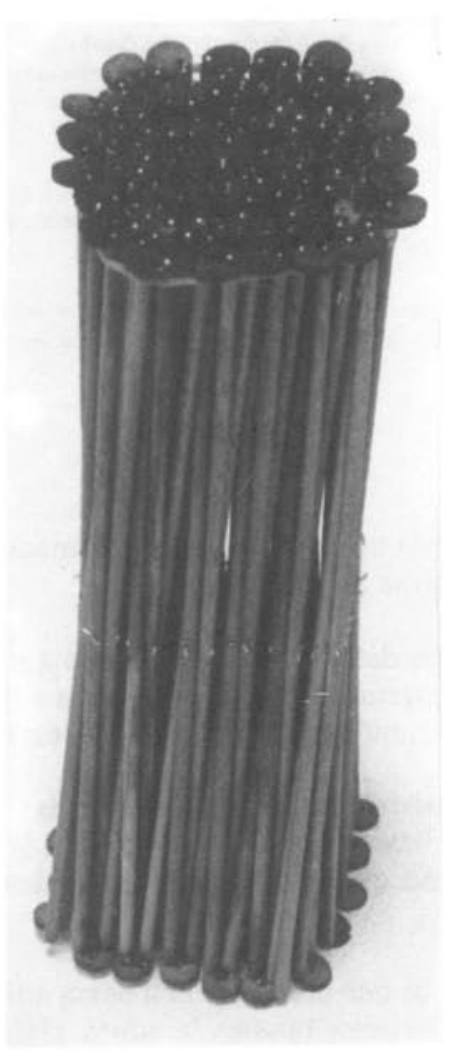




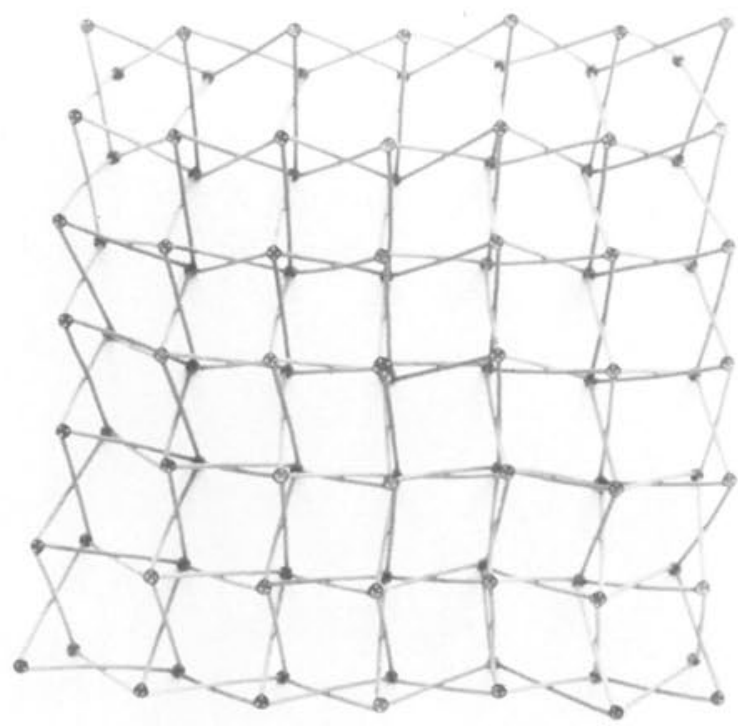

Fig. 8

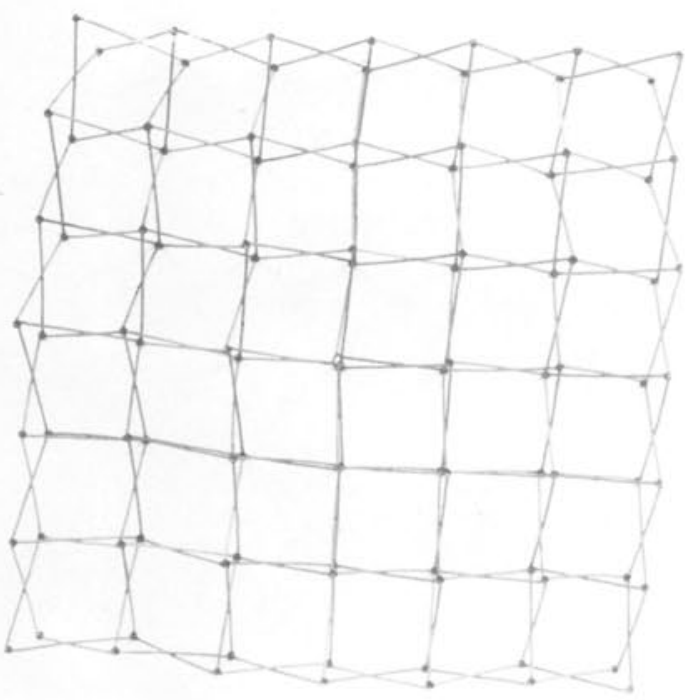

Fig. 9

mensión se moverá el conjunto. Preparando un conjunto complejo de elementos que se crucen a $60^{\circ}$ obtenemos estructuras como la que se ilustra en tres estados distintos en las figuras 11,12 y 13 .

En lugar de cruzar los elementos cortándose en una linea vertical, podemos hacerlo oblicuamente según un modelo representado en la figura 14 y si cada uno de estos cuatro planos es una sucesión de aspas, como la de la figura 1, habremos obtenido un conjunto plegable del mismo modo que el elemento base.

Con este sistema de encuentro podemos elaborar estructuras más complejas como la representada en tres estados distintos en las figuras 15,16 y 17 .

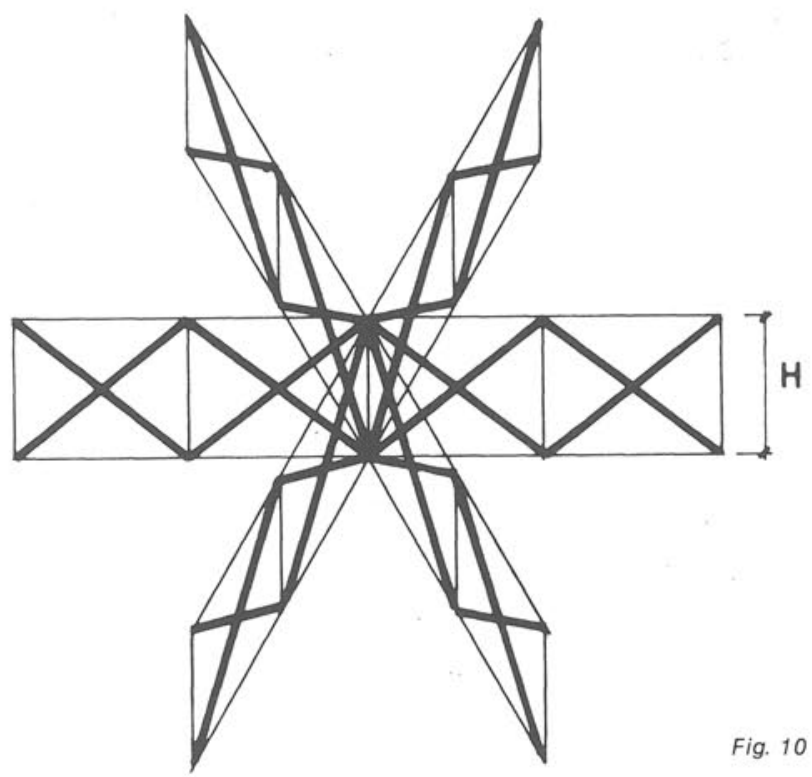

Fig. 11

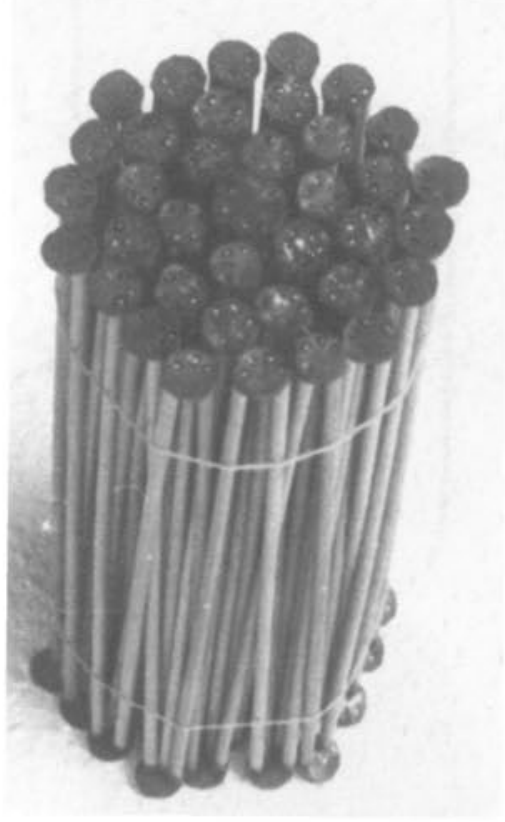

Fig. 12

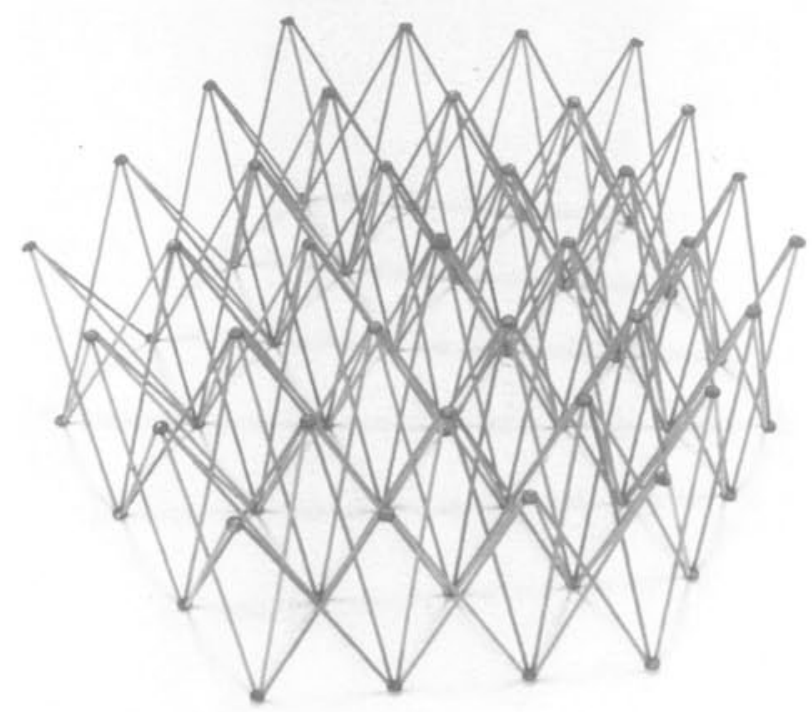




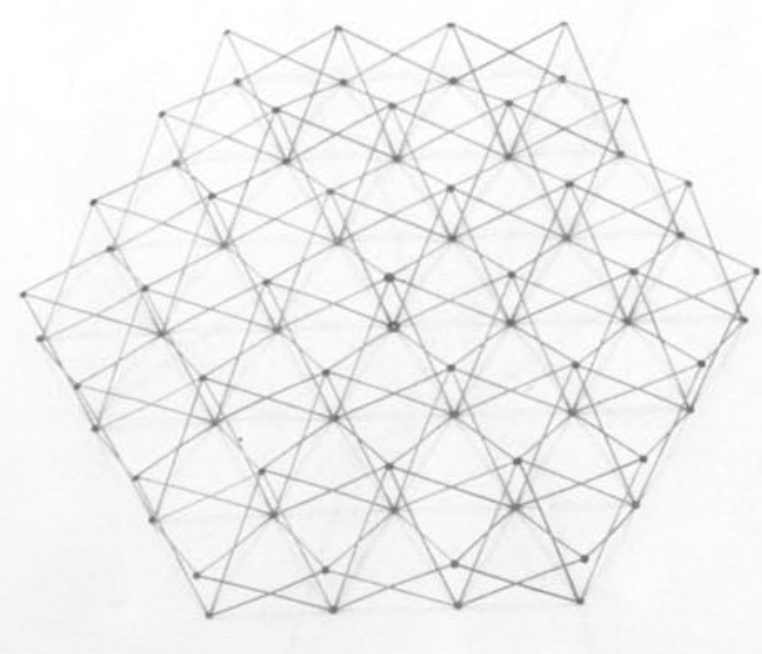

Fig. 13

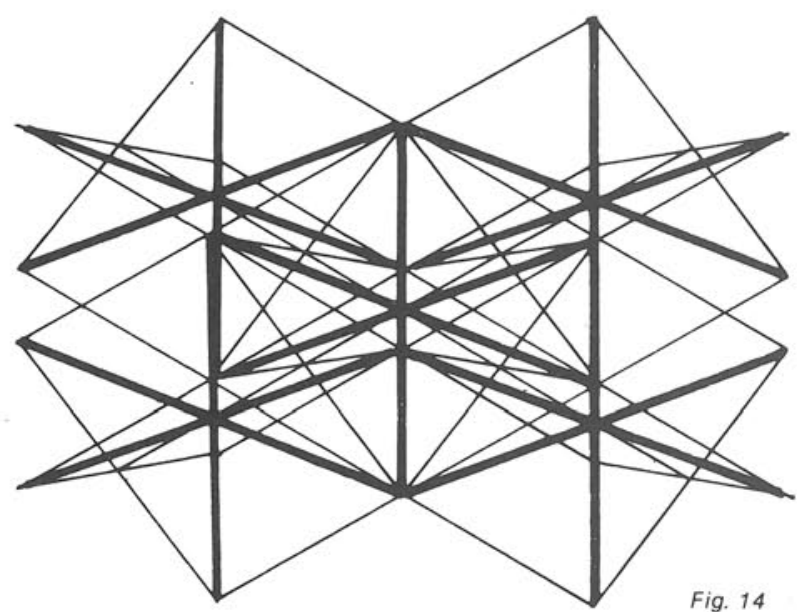

Fig. 14

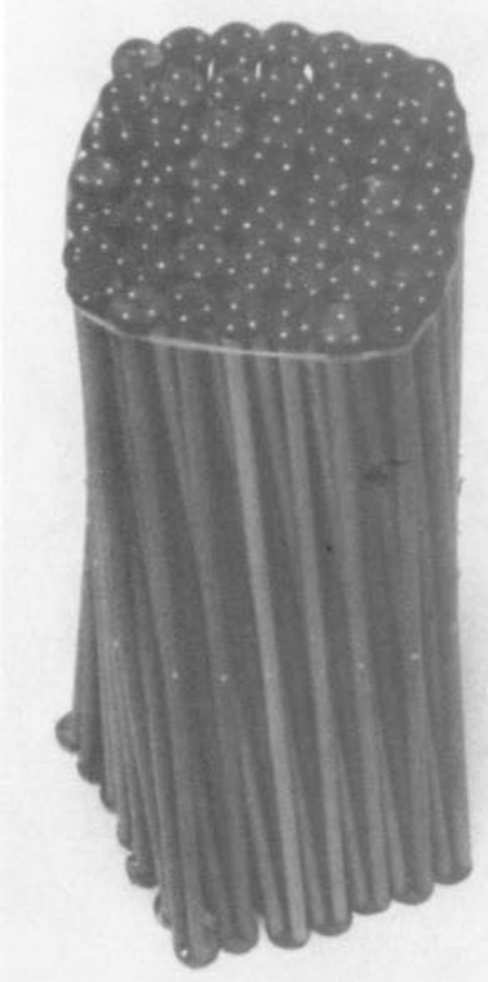

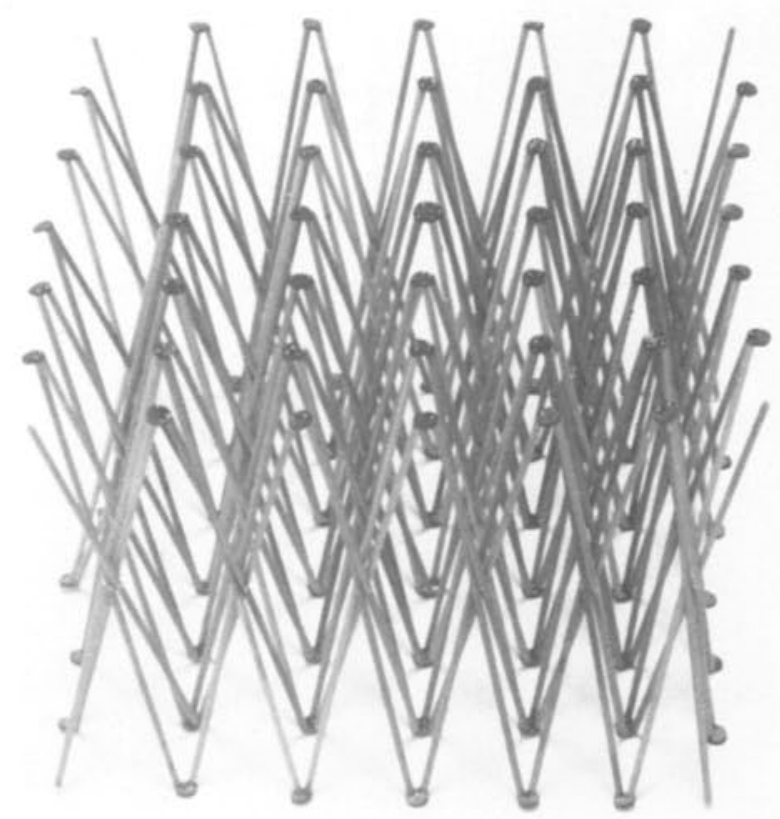

Fig. 16

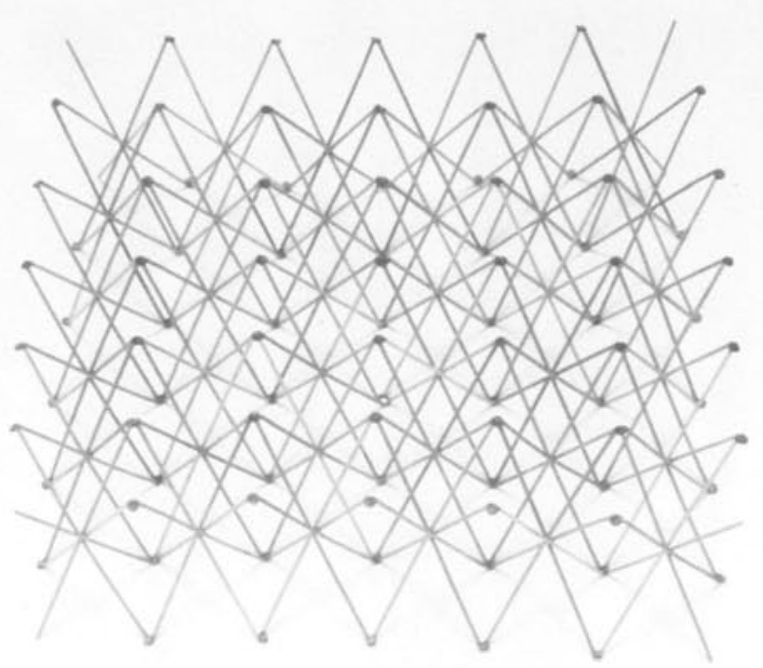

Fig. 17

Otra forma básica de ensamble es la expresada en la figura 18, que da lugar a estructuras como la representada en las figuras 19, 20 y 21 en tres fases de plegado.

Hay más posibilidades de conexión y por tanto más tipologías posibles de estructuras desplegables de barraś, por ejemplo, cortando en cada linea más planos elementales. Pero para una modulación espacial continua éstas son las básicas.

A partir de los cuatro sistemas de conexión descritos podemos generar una enorme variedad de estructuras con contornos distintos y esquemas de sustentación muy diferentes. Algunos de ellos se muestran en las figuras procedentes para sistemas planos de crecimiento en superficie. 

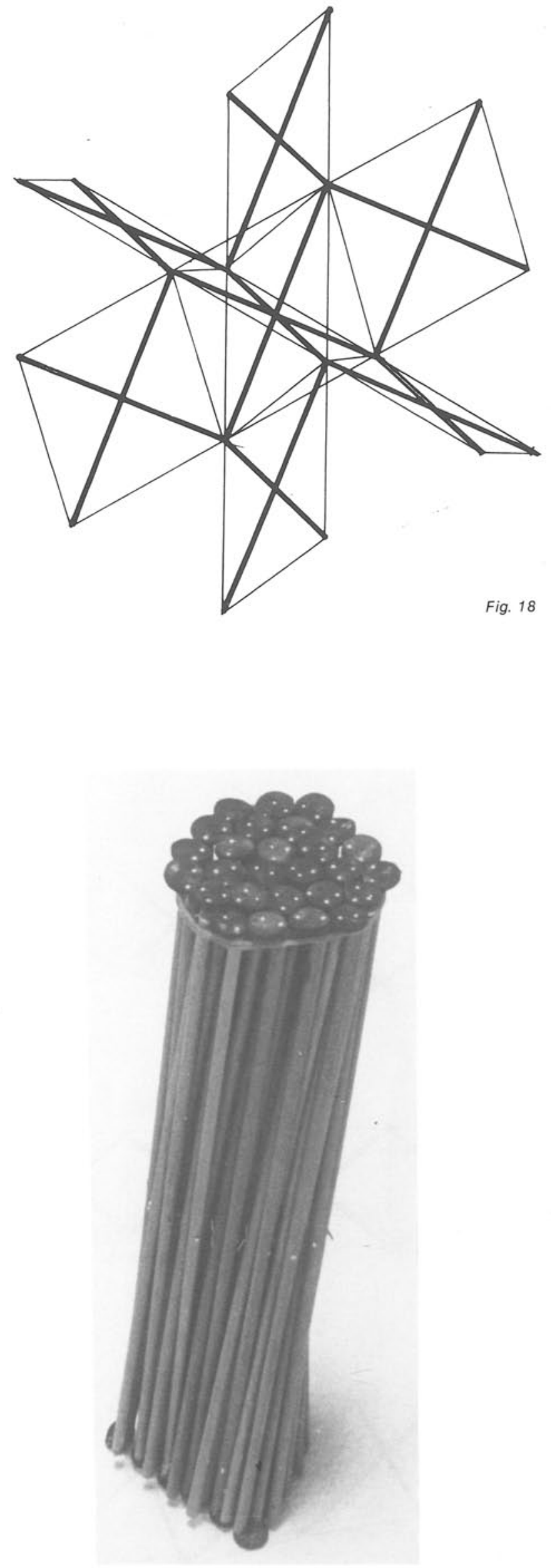

Fig. 19

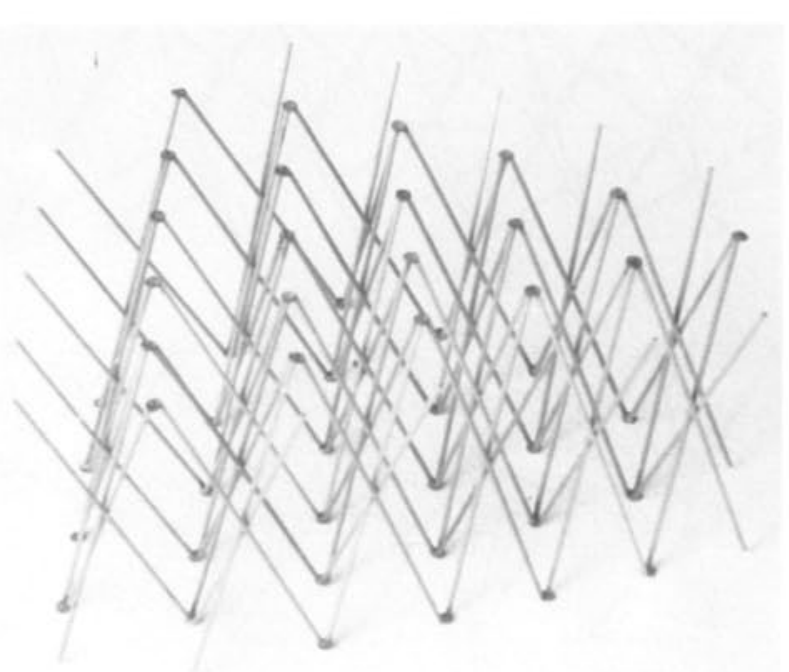

Fig. 20

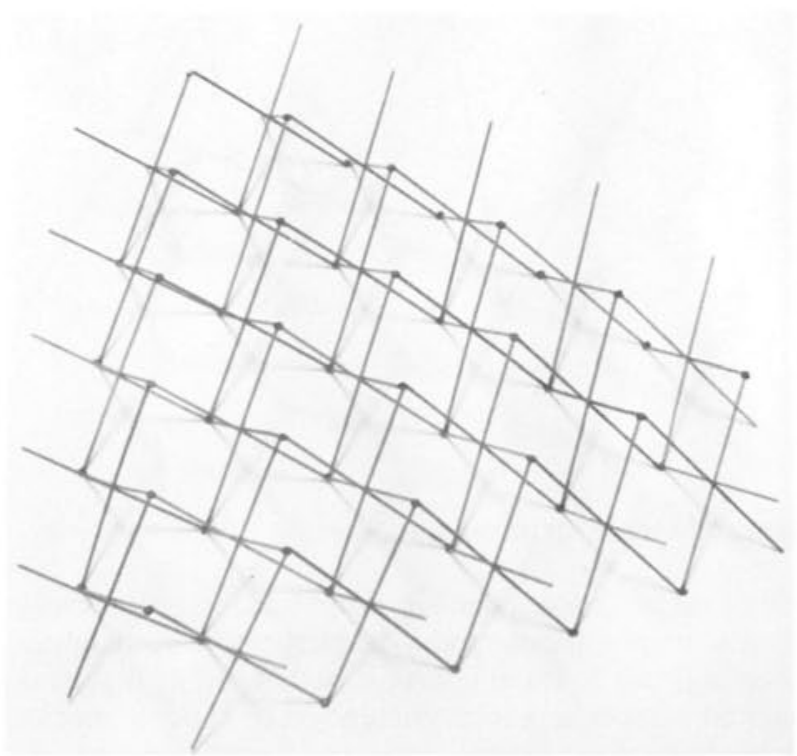

Fig. 21

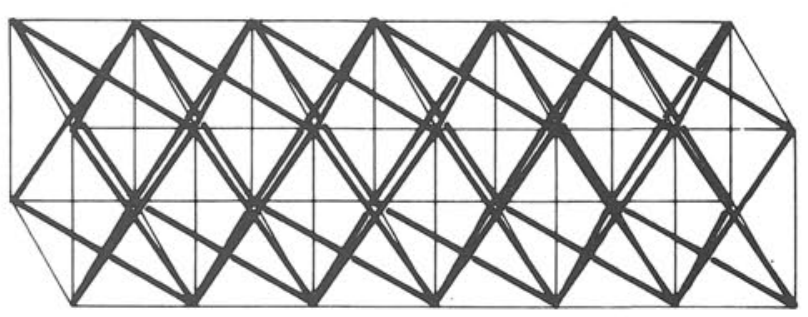

Fig. 22

El crecimiento puede ser también tridimensional superponiendo varios niveles de los descritos. Y puede ser también longitudinal procurando una ordenación con una sola dirección de crecimiento (Figs. 22, 23, 24 y 25).

Con estos esquemas podemos crear conjuntos mixtos en función de distintos diseños. 


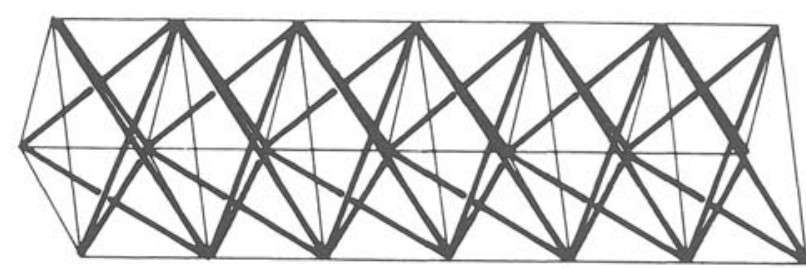

Fig. 23

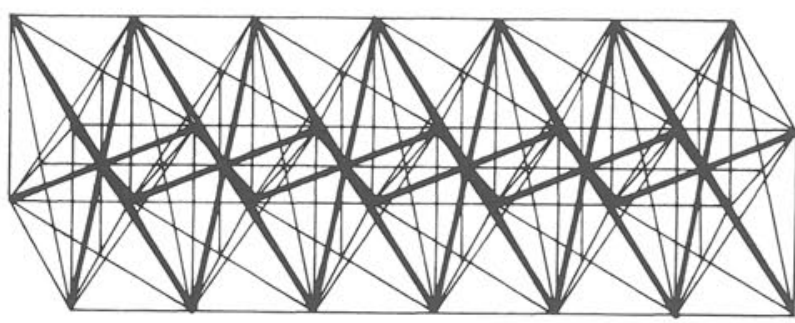

Fig. 24

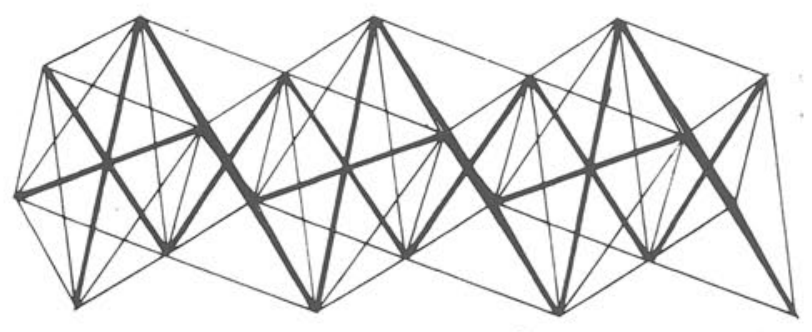

Fig. 25

\section{Generalización del sistema}

Vamos ahora a extender las posibilidades geométricas del sistema por degeneración del elemento básico definido en la figura 1. En él todas las barras son de la misma longitud y tienen una sola articulación en el punto medio. Podemos cambiar ambos supuestos separada o simultáneamente para obtener elementos básicos también desplegables pero con propiedades bastante diferentes:

a) Por ejemplo, podemos utilizar barras de la misma longitud y con la articulación interior excéntrica (Fig. 26). Como vemos, el conjunto se curva circularmente si la proporción 1/1' es constante, y con curvatura variable si la proporción $1 / 1$ ' es variable. La única condición que se impone es que $1+1^{\prime}=$ cte en todas las barras.

b) Si utilizamos barras de distinta longitud con la articulación centrada, obtendremos esquemas como el de la figura 27, que utilizan sólo dos tipos de barras o como el de la figura 28 si se utilizan barras de muchos tamaños. En este caso la única condición necesaria será que $1_{\mathrm{i}}+1_{\mathrm{i}}^{\prime}=$ cte.

c) Si utilizamos simultáneamente barras de distinta longitud y articulación excéntrica, podemos obtener una gran variedad de tipos de los que se muestran algunos en las figuras 29,30 ó 31 .

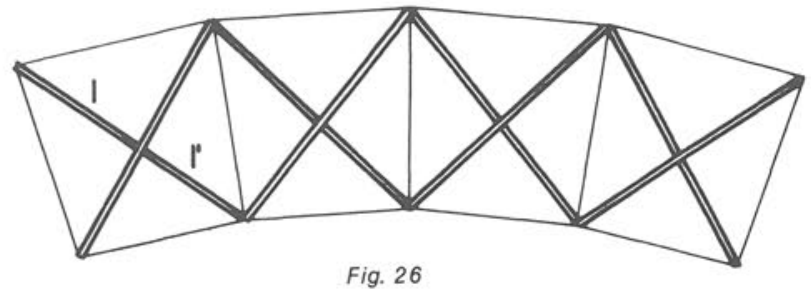

Fig. 26

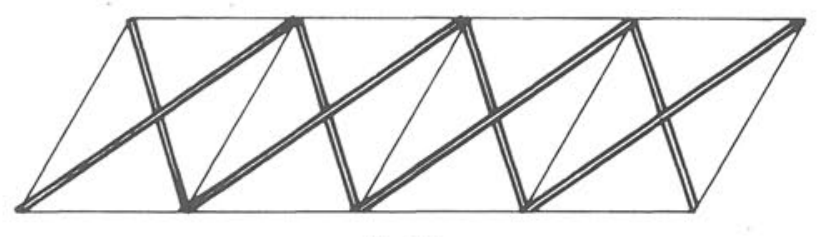

Fig. 27

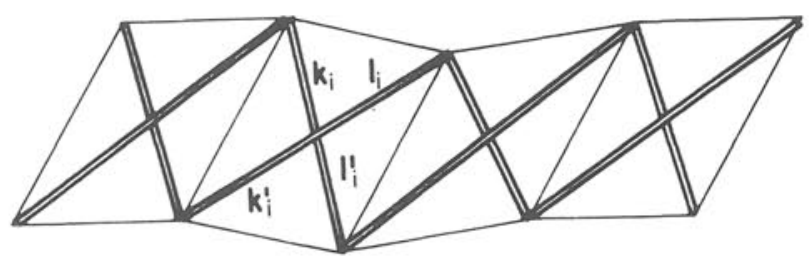

Fig. 28

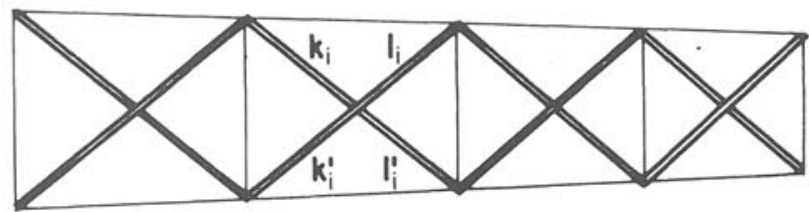

Fig. 29

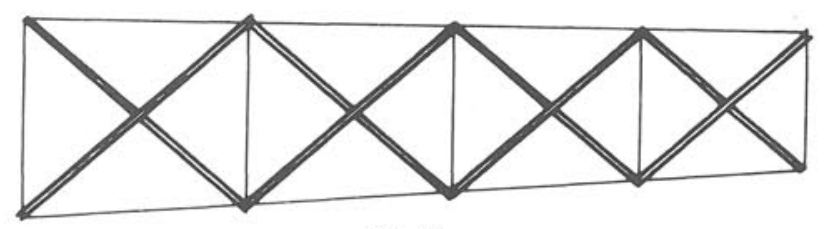

Fig. 30

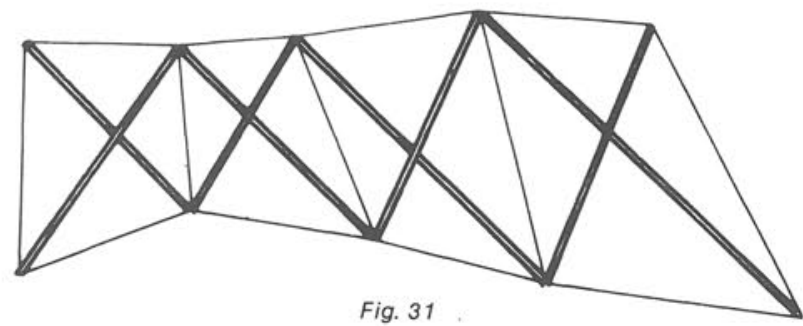

http://informesdelaconstruccion.revistas.csic.es 
La condición necesaria y suficiente para que el plegado sea posible es que en todas las aspas $1_{\mathrm{i}}+1_{\mathrm{i}}^{\prime}=\mathrm{k}_{\mathrm{i}+1}+$ $+\mathrm{k}_{\mathrm{i}+1}^{\prime}$.

d) Pueden utilizarse sistemas de aspas de más de una capa con las mismas variantes anteriores (Figs. 32, $33,34$ y 35$)$.

Y la condición que deben cumplir los tramos de barra es bastante más compleja en los casos más irregulares.

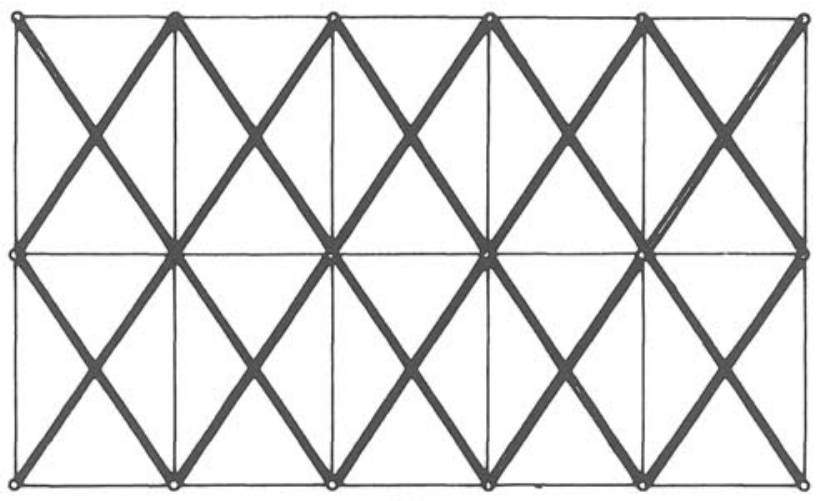

Fig. 32

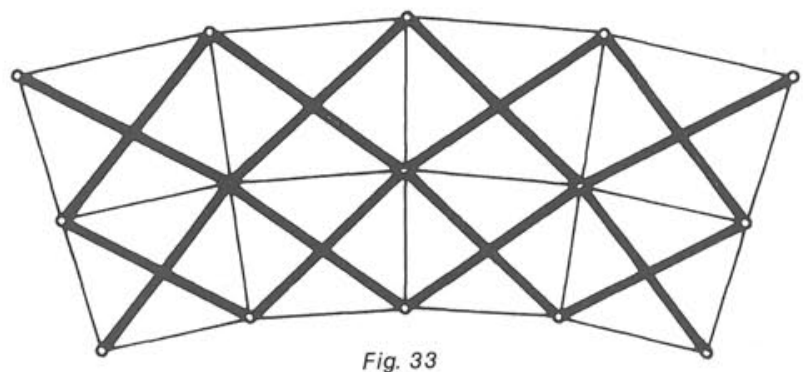

Fig. 33

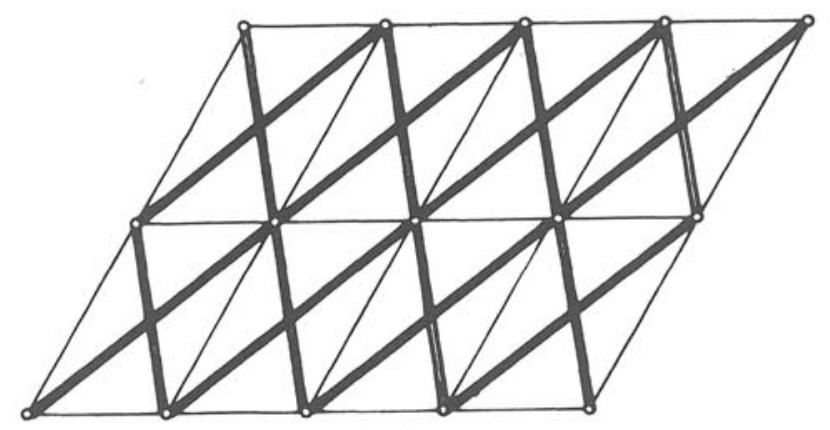

Fig. 34

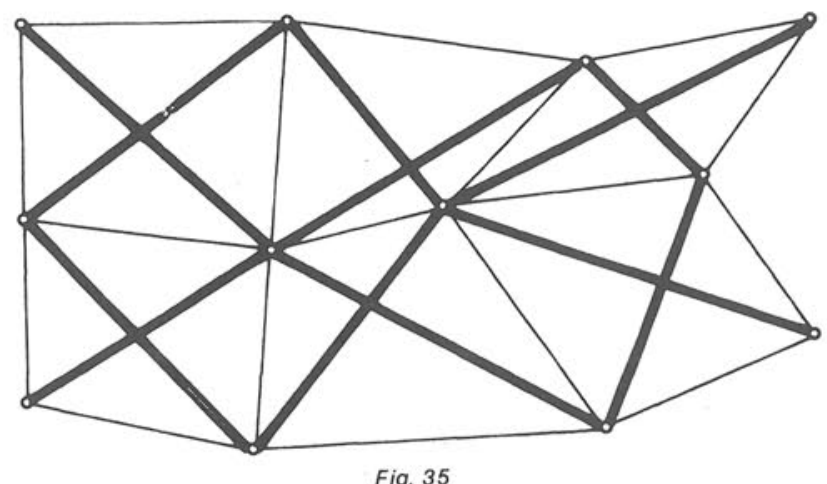

Fig. 35

\section{Aplicaciones prácticas}

Las posibilidades de diseño a partir de la casuistica descrita son enormes y se aportan algunos casos a titulo de ejemplo.

En la figura 36 se representa una sombrilla plegable hexagonal plana, con modulación triangular, apoyada en su mástil central.

En la figura 37 se representa una cubierta cilindrica apoyada en dos de sus generatrices rectas, a partir de una modulación rectangular.

En la figura 38 se representa un arco apoyado en dos extremos, a partir de una modulación triangular.

En la figura 39 se representa una cúpula esférica apoyada en cuatro esquinas, a partir de una modulación cuadrada.

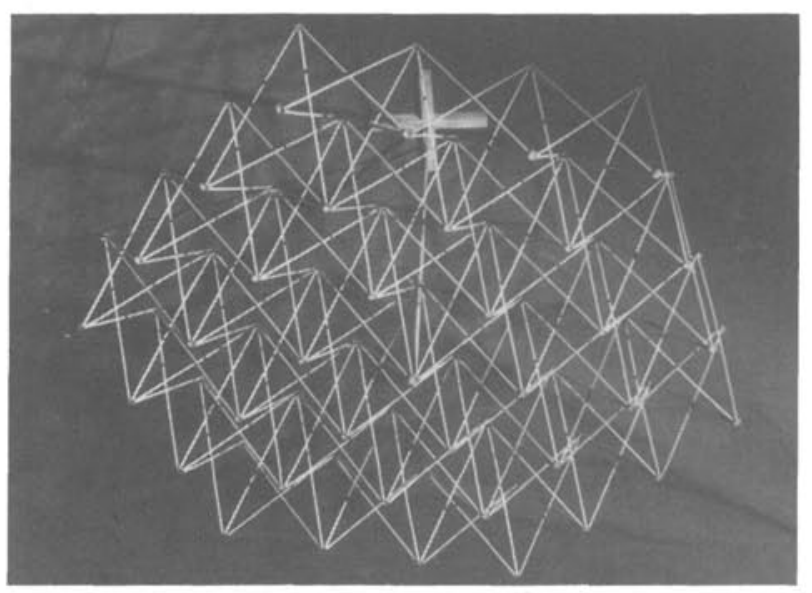

Fig. 36

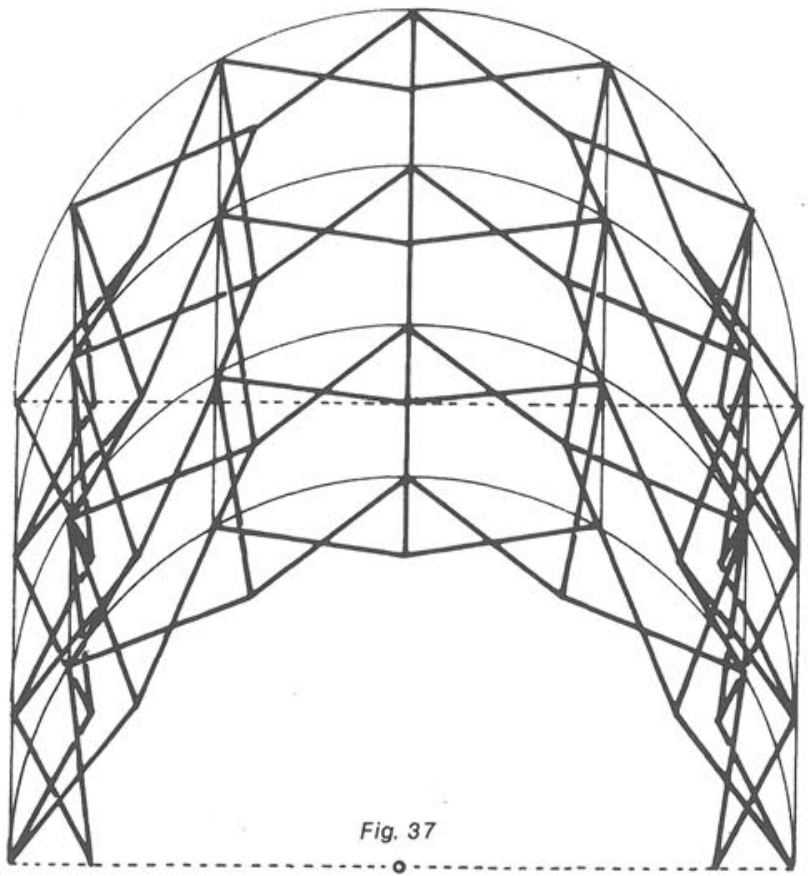


En la figura 40 se representa una sombrilla piramidal apoyada en un mástil central con evacuación de aguas hacia el interior.

La figura 41 representa una cúpula hemisférica construida con barras de la misma longitud.

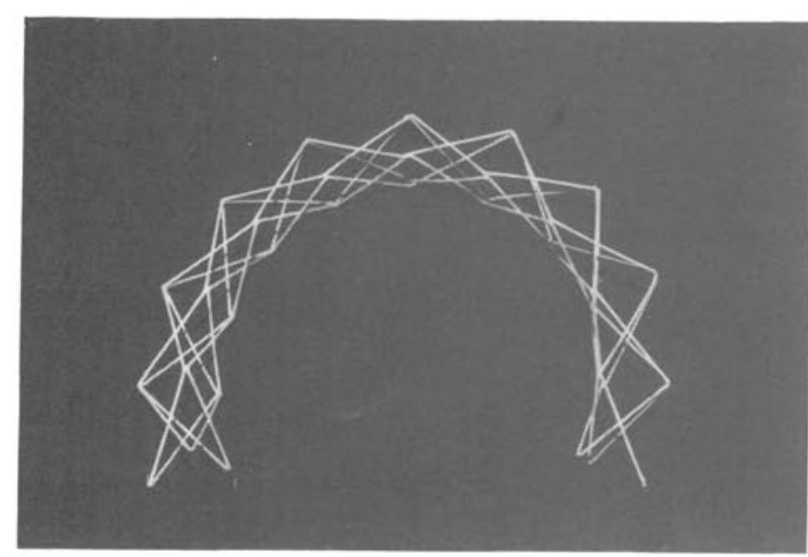

Fig. 38

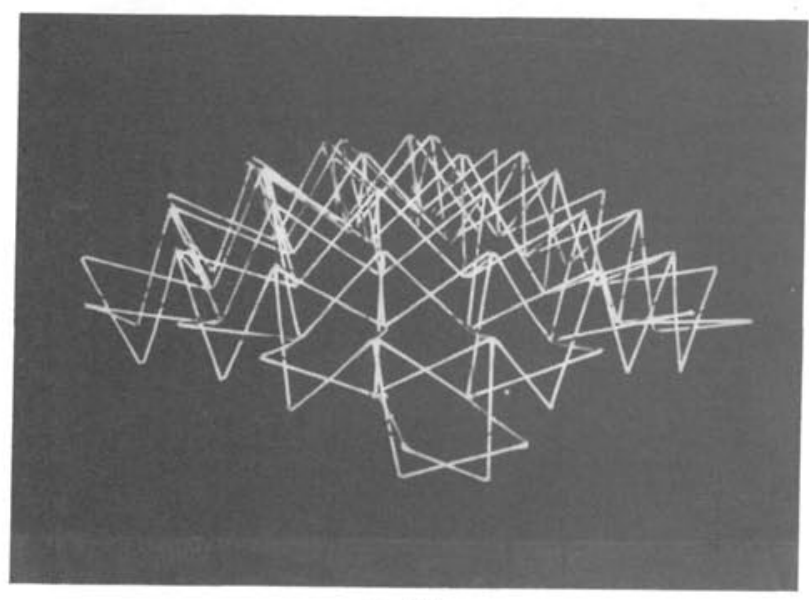

Fig. 39

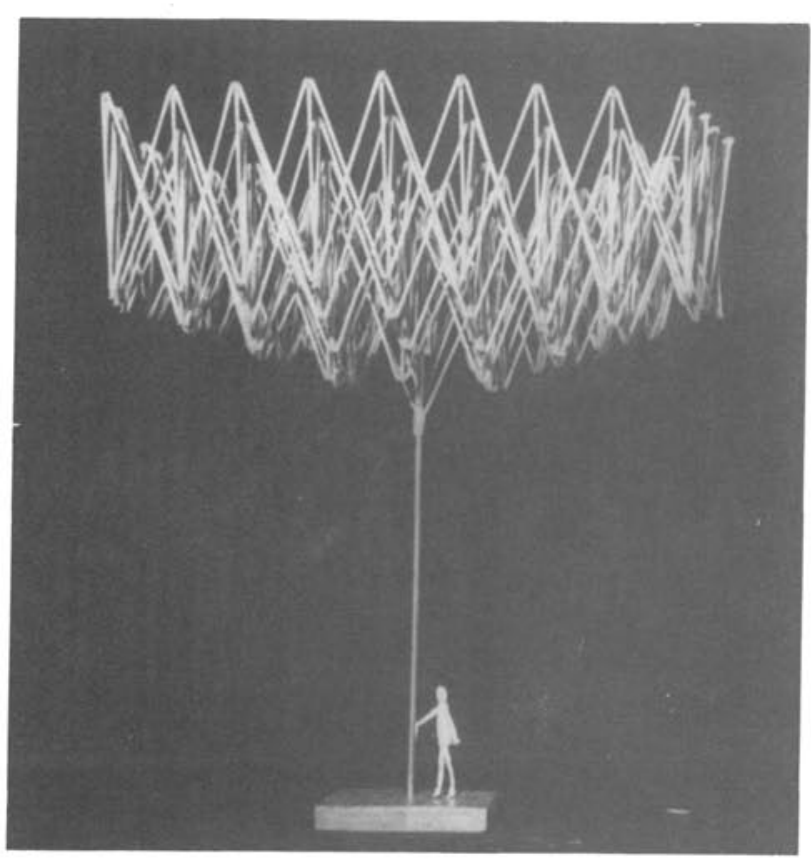

Fig. 40
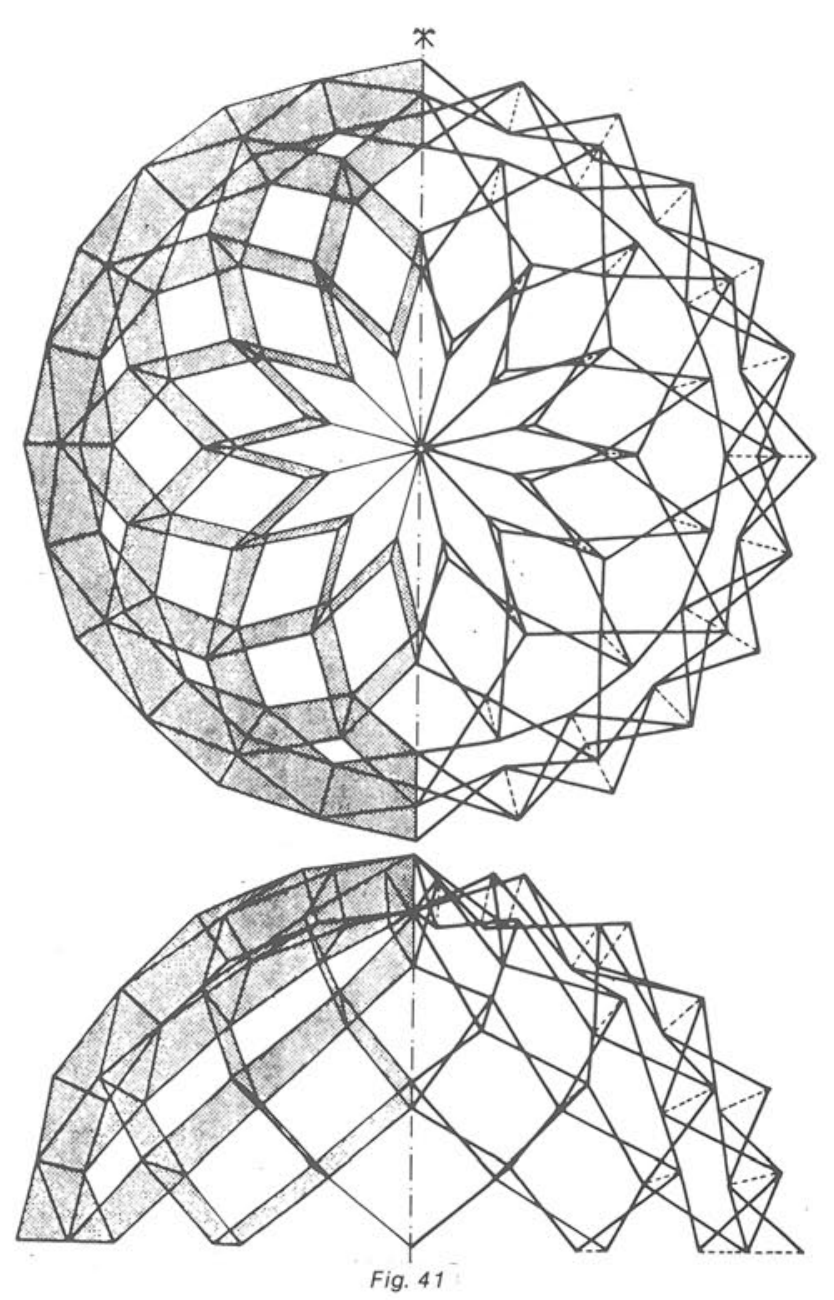

\section{Aspectos arquitectónicos}

Para su utilización en empresas arquitectónicas estas estructuras pueden utilizarse con su propio valor escultórico como exhibición de conjuntos móviles. Pero con carácter funcional, en coberturas o cerramientos, hay que acompañarlas de un elemento de cobertura que lo haga útil como protección del sol o de la lluvia y que puede consistir en alguna lona que, sujeta a los nudos superiores o inferiores, puede plegarse junto a las barras y desplegarse para dar una superficie continua y tensa que puede ayudar a rigidizar las estructuras, algunas de las cuales necesitan dotarse de una rigidización suplementaria frente a deformaciones angulares.

Si esta capa textil es doble, una por la cara superior y otra por la inferior, puede conseguirse una cámara térmica que mejore la protección del conjunto (Fig. 42).

Estas lonas pueden dotarse de elementos auxiliares que fuercen su plegado ordenado, como se expresa en la figura 43, en que unos cables de longitud "m» que unen puntos centrales del tejido, al mantener su longitud, introducirán la tela hacia el interior, facilitando el plegado (Fig. 44). 


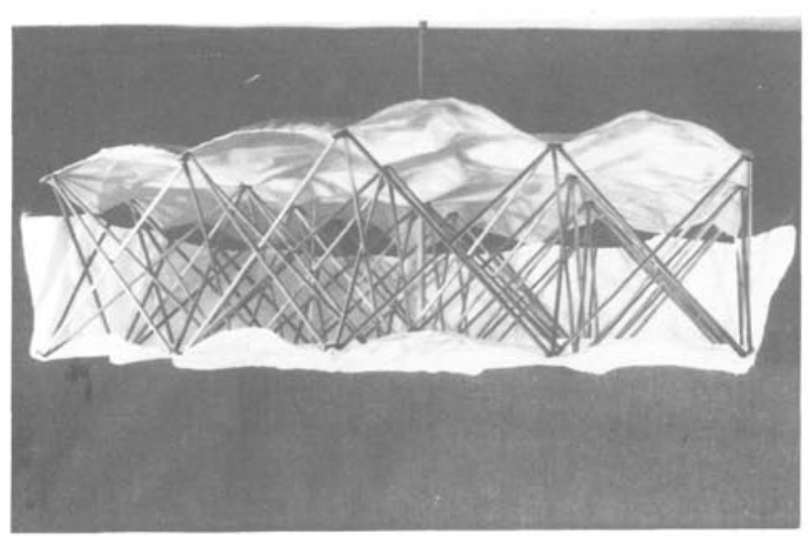

Fig. 42

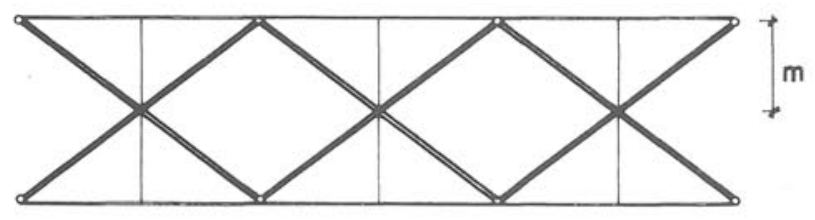

Fig. 43

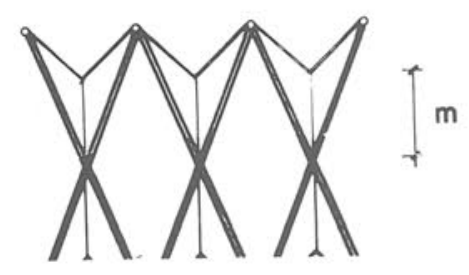

Fig. 44

\section{Aspectos constructivos}

Como en las estructuras espaciales convencionales de barras, y en estos casos con mucha más razón, la buena solución de los nudos es la base del funcionamiento eficaz del conjunto para que realmente sea posible el plegado y desplegado. En las figuras 45 a 54 se ilustran algunas soluciones particulares utilizadas en diseños proyectados y maquetas realizados, mientras que en las figuras 55 a 61 se definen algunos sistemas de unión de estos nudos a las barras que articulan. El número de encuentros depende de cada solución particular.

Un hecho que facilita el diseño de estos nudos es que la mayor parte de los movimientos de las barras se producen en un plano determinado, con lo cual sólo es necesario garantizar el giro en él y no es necesario recurrir a costosas articulaciones espaciales.

Otro de los aspectos fundamentales a resolver es el de los procedimientos mecánicos de plegado y desplegado. En las figuras 62,63 y 64 se describen tres soluciones particulares con buen resultado práctico.

En la figura 62 un motor "A» ligado a un elemento de unión « $\mathrm{B} »$ acciona un tornillo « $\mathrm{C} »$ que fuerza el acercamiento de una tuerca "E» ligada a un elemento de unión «D» situado en el extremo opuesto. El conjunto de las estructuras puede estar provisto de uno o varios de estos

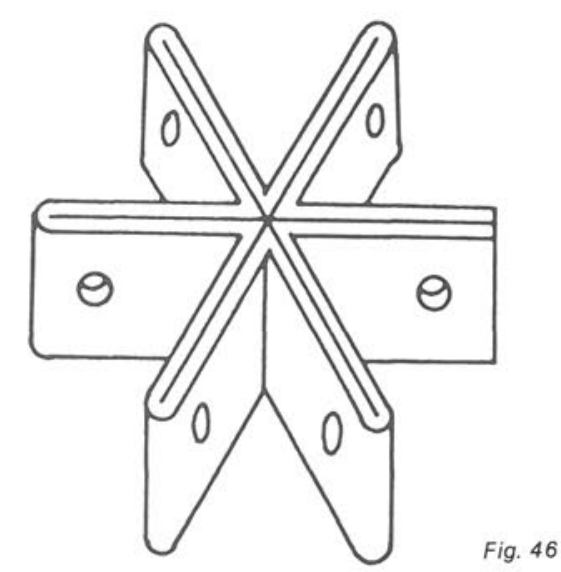

Fig. 46
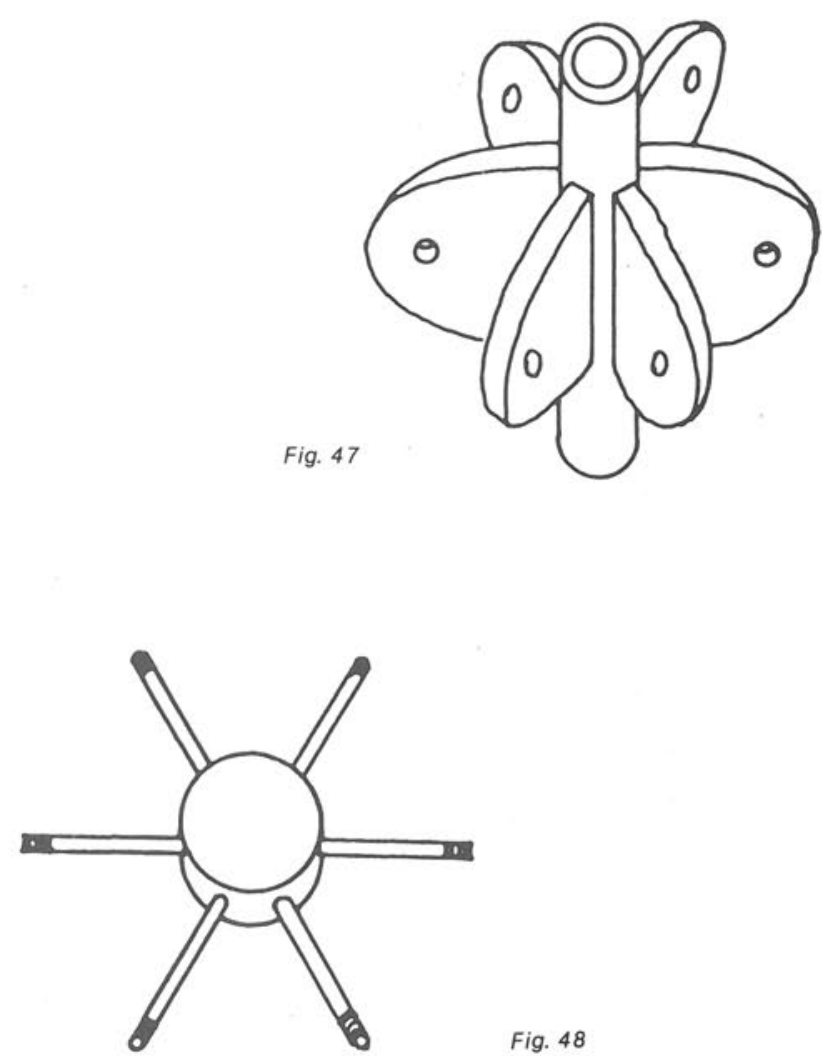

Fig. 48 

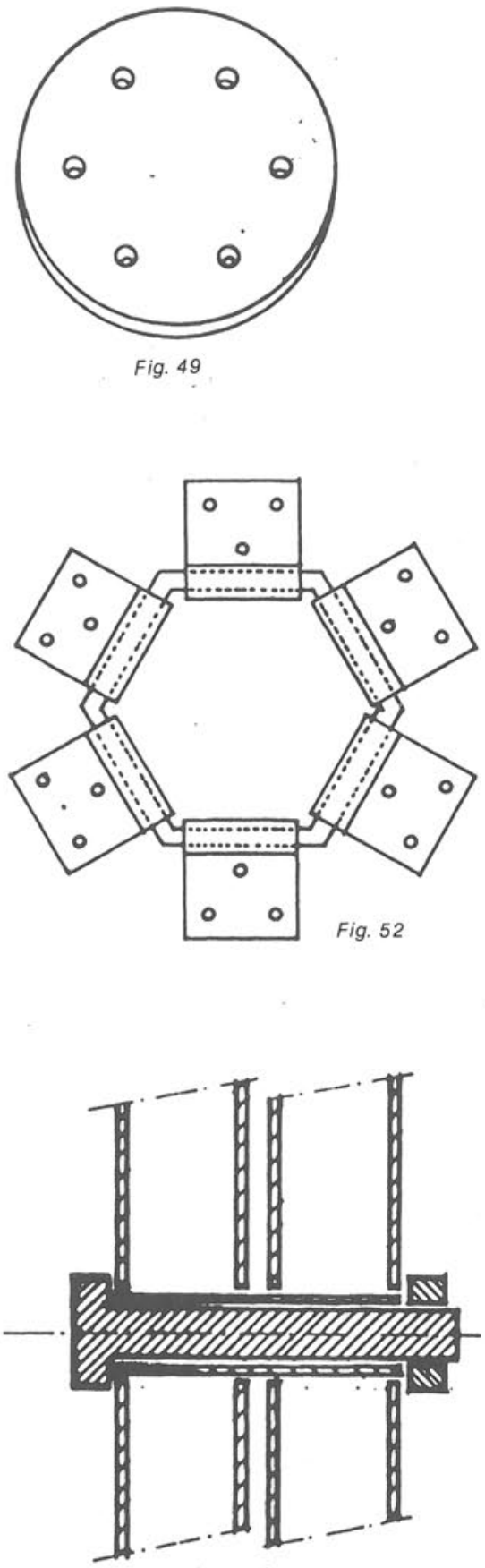

Fig. 55

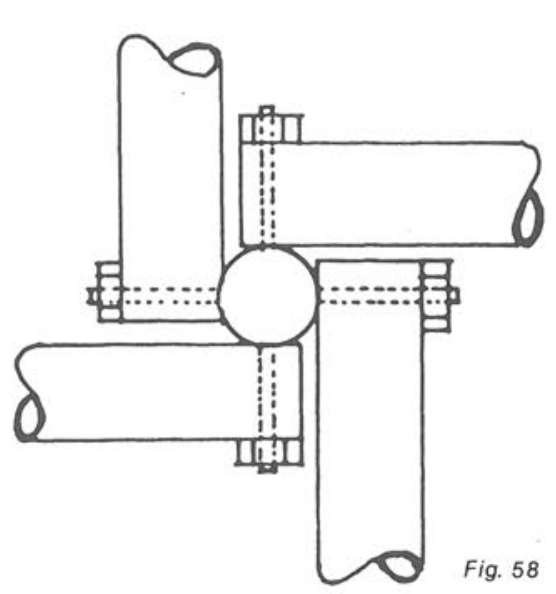

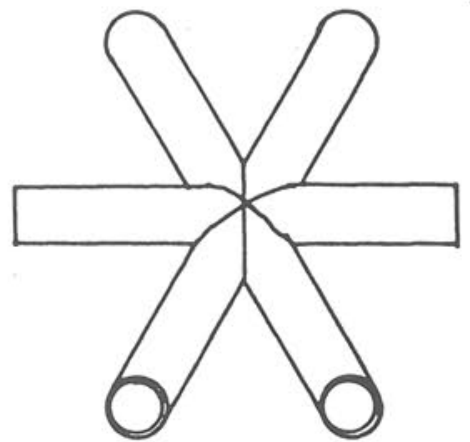

Fig. 50
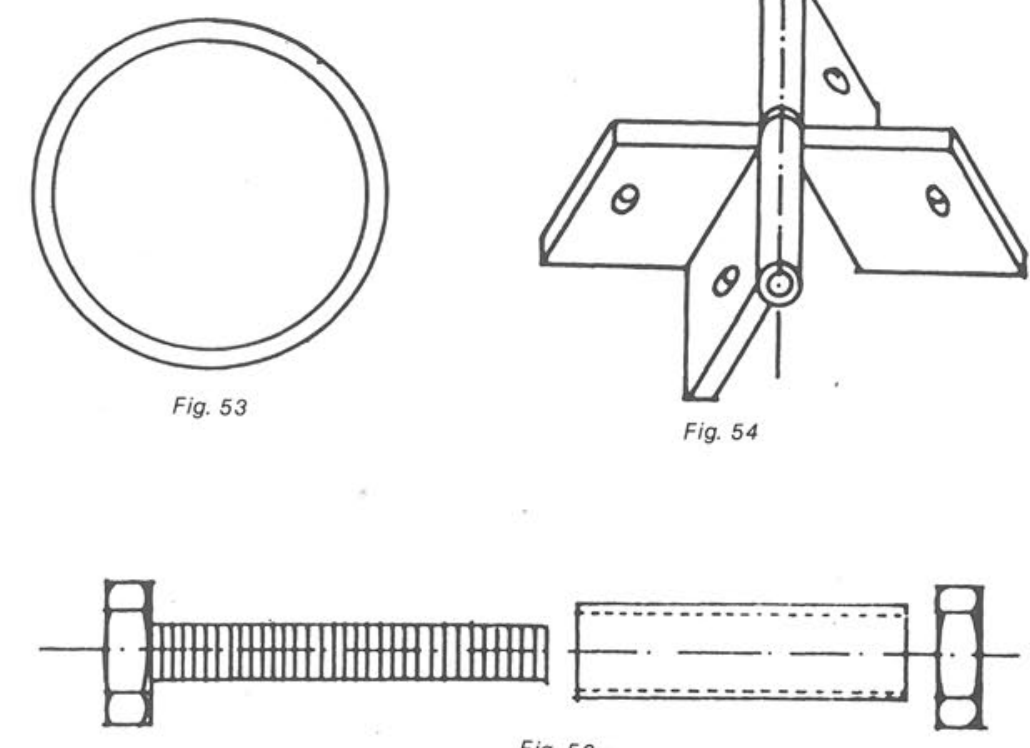

Fig. 56
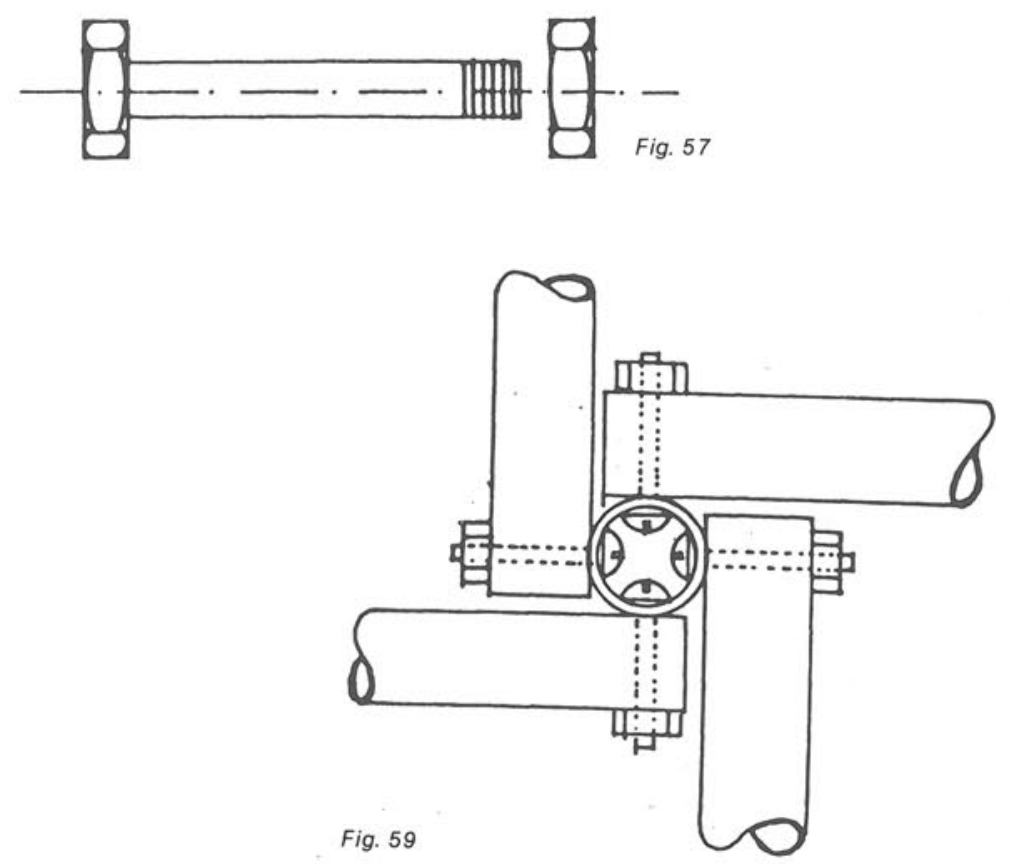

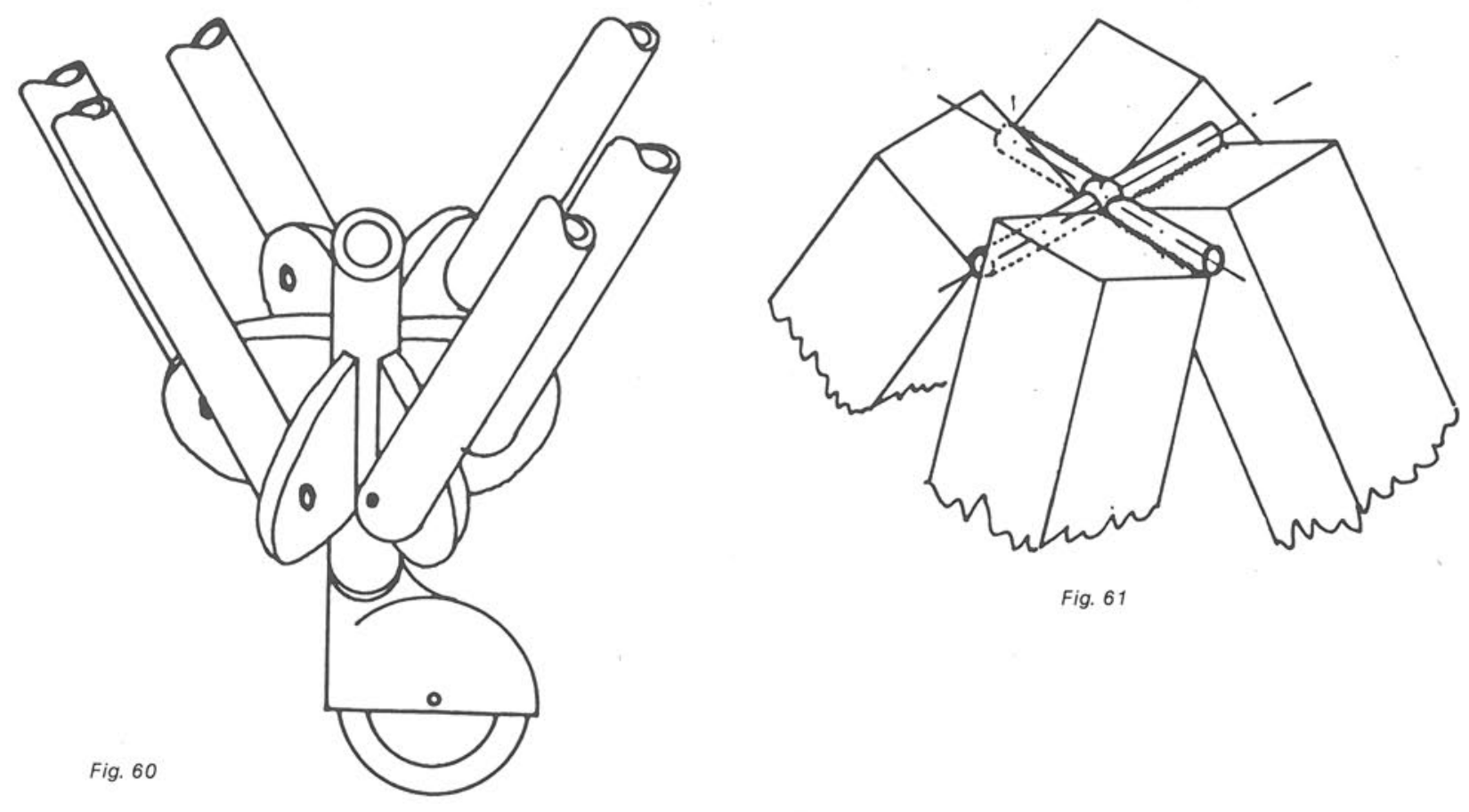

Fig. 61
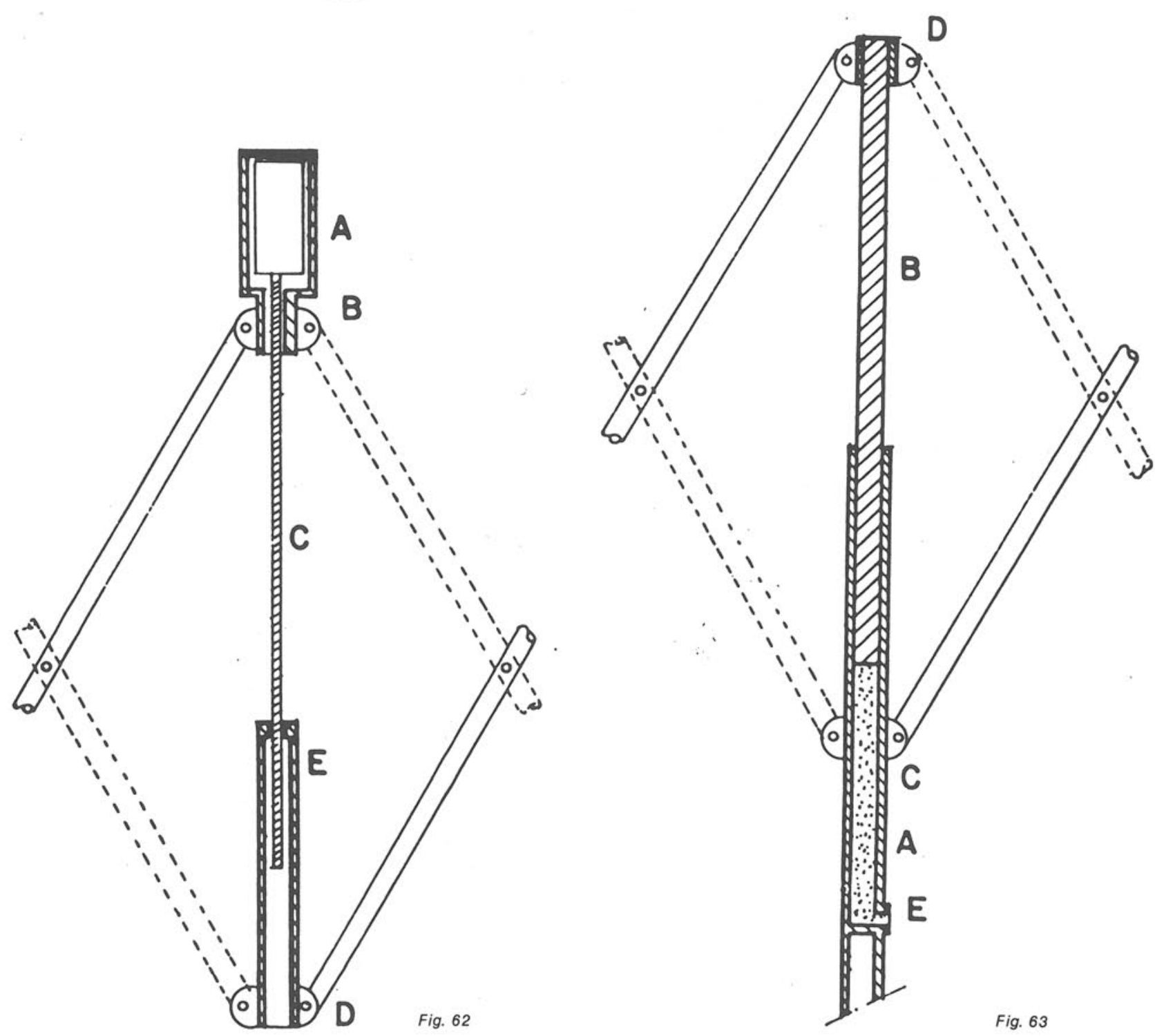


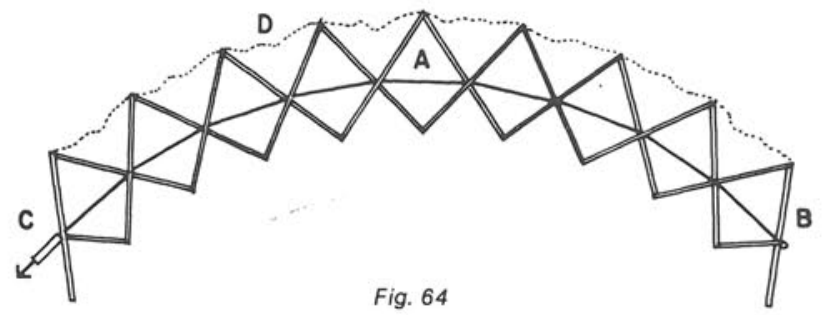

elementos para que puedan ser más ligeros y que las acciones de plegado y desplegado se distribuyan mejor entre todas las barras.

En la figura 63 se describe un sistema hidráulico ligado a conjuntos con mástil central. El émbolo del gato «B» es el que propicia la separación entre los nudos «C» $\mathrm{y}$ " $\mathrm{D} »$, mientras que su acercamiento se consigue por gravedad al dejar de actuar la presión hidráulica.

En la figura 64 se describe un procedimiento de plegado que consiste en forzar el acercamiento de nudos muy alejados, mediante un cable con un elemento tensor. El desplegado en este caso puede conseguirse también por gravedad.

\section{Particularidades de cálculo}

Los aspectos de cálculo pueden ser complejos en estas estructuras de elevado número de barras articuladas, en las que los esfuerzos de flexión son protagonistas del comportamiento resistente, y ninguno de los programas de ordenador convencionales está capacitado para considerar nudos articulados y empotrados selectivamente para según qué barras consideremos en ellos. El Departamento de Estructuras de la Escuela de Arquitectura de Sevilla trabaja en ello y este tema será objeto de próxima publicación.

En cuanto al predimensionado es sencillo sin más que considerar el sistema de generación del conjunto de cada estructura particular.

En estructuras simétricas de forma y carga, el dimensionado previo puede reducirse al cálculo de estructuras como las de la figura 65 .

En la figura 66 se expresan los diagramas de momentos flectores:

$$
\begin{aligned}
& \mathrm{M}_{1}=\mathrm{P}_{1} \cdot 1 ; \mathrm{M}_{2}=\left(\mathrm{P}_{1}+\mathrm{P}^{2}\right) \cdot 1 ; \\
& \mathrm{M}_{3}=\left(\mathrm{P}_{1}+\mathrm{P}_{2}+\mathrm{P}_{3}\right) \cdot 1
\end{aligned}
$$

En la figura 67 los diagramas de esfuerzos cortantes:

$$
\begin{gathered}
\mathrm{Q}_{1}=\mathrm{P}_{1} \operatorname{sen} \alpha \quad \mathrm{Q}_{2}=\left(\mathrm{P}_{1}+\mathrm{P}_{2}\right) \operatorname{sen} \alpha \\
\mathrm{Q}_{3}=\left(\mathrm{P}_{1}+\mathrm{P}_{2}+\mathrm{P}_{3}\right) \operatorname{sen} \alpha
\end{gathered}
$$
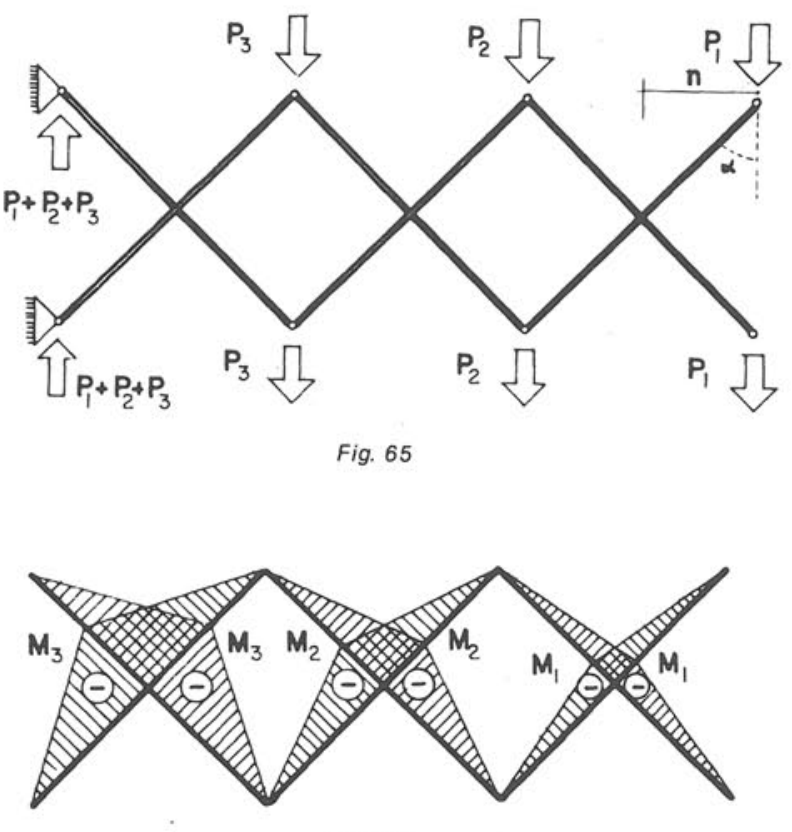

Fig. 66

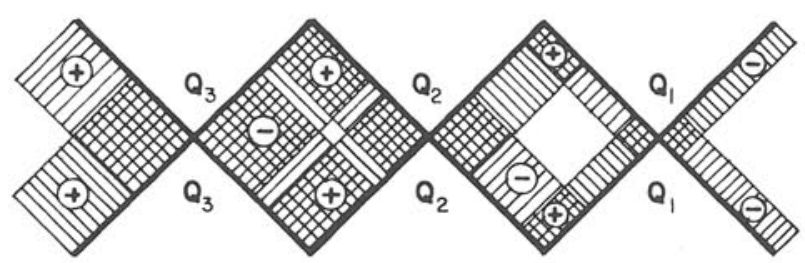

Fig. 67

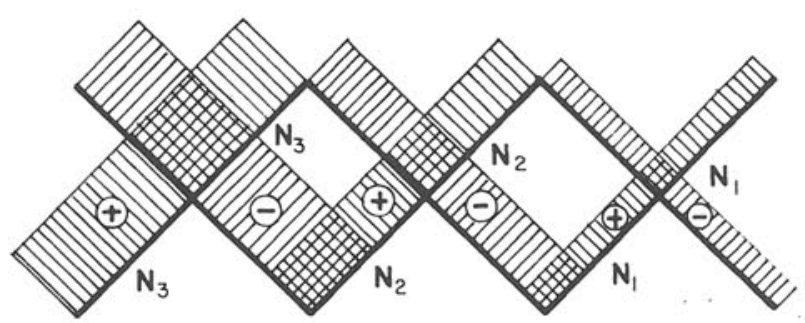

Fig. 68

En la figura 68 los diagramas de esfuerzos axiles:

$$
\begin{gathered}
\mathrm{N}_{1}=\mathrm{P}_{1} \cos \alpha \quad \mathrm{N}_{2}=\left(\mathrm{P}_{1}+\mathrm{P}_{2}\right) \cos \alpha \\
\mathrm{N}_{3}=\left(\mathrm{P}_{1}+\mathrm{P}_{2}+\mathrm{P}_{3}\right) \cos \alpha
\end{gathered}
$$

La rigidez a torsión de las aspas utilizadas puede tener influencia importante en la distribución de esfuerzos sobre todo en estructuras muy asimétricas.

Cuando la capa de tejido adicional sea considerada con su resistencia a tracción se introduce otro grado de complejidad en el cálculo pero se mejora el comportamiento resistente del conjunto. 